INTERNATIONAL JOURNAL OF RESEARCHES IN BIOSCIENCES, AGRICULTURE \& TECHNOLOGY ( ) VISHWASHANTI MULTIPURPOSE SOCIETY (Global Peace Multipurpose Society) R. No. MH-659/13(N) www.vmsindia.org

\title{
ASSOCIATIVE AND ANTAGONISTIC EFFECT OF FUNGI FROM PHYLLOSPHERE AND FRUIT OF BANANA.
}

\author{
Mahadik S. C.
}

Ramkrishna Paramhansa Mahavidyalaya, Osmanabad (M.S.) India

Email: drmahadiksc@rediffmail.com

\begin{abstract}
Economic value of fruits are higher however post harvest loss to fruits is 5 to $50 \%$ or even more due to lack of awareness at all stages from fruit production to fruit consumption. During ordinary storage and transit a single fruit can spread the infection in the whole lot. Banana is popular fruit due to its nutritive value. However banana suffers from many pre and post harvest diseases . In post harvest disease Fusarium oxysporum was commonly found on many samples. Sensitivity of pathogen was tested against fungicides.Now a days integrated management of disease is emphasized. A total of fifteen fungal species isolated from banana fruits and phyllosphere were studied on agar by spot inoculation method for their associative and antagonistic effect.Various associative effects were seen. Aspergillus species did not show any harmful effect against each other. Tricoderma species showed antagonistic zone towards Fusarium oxysporum. Inhibitory zone shows that fungal culture filtrate was more useful for management of pathogen.
\end{abstract}

Keywords: Banana, Fusarium, Trichoderma

\section{Introduction:}

Post harvest diseases are those that develop during harvesting, grading, packing and transportation or at the market and during the various handling operations required to move the crop from grower to consumer. In India banana

(Musa paradisica L.) is a popular fruit due to its nutritive value. However banana suffer from many post harvest diseases .In present study antagonistic effect of fungi from phyllosphere and fruit of banana is studied.

\section{Material and Method:}

The diseased samples of banana were collected from different localities .The pathogen Fusarium oxysporum was isolated from the infected tissue by using czapak Dox agar medium. The sensitivity of isolates was tested against benomyl. By EMS treatment benomyl resistant mutant (EMSFo4) was obtained. A total of 15 fungal species isolated from phyllosphere and fruits of banana were tested against Fusarium oxysporum resistant mutant (EMSFo 4) on agar by spot inoculation method.

\section{Result:}

Table-1 : Phyllophere fungi / fruit fungi of banana

\begin{tabular}{|c|l|c|}
\hline $\begin{array}{c}\text { Sr } \\
\text { No }\end{array}$ & \multicolumn{1}{|c|}{ Fungi } & Colonies \\
\hline 1 & Aspergillus flavus & 12 \\
\hline 2 & Aspergillus niger & 2 \\
\hline 3 & Aspergillus fumigatus & 7 \\
\hline 4 & Aspergillus terrus & 3 \\
\hline 5 & $\begin{array}{l}\text { Aspergillus } \\
\text { carbonarius }\end{array}$ & 4 \\
\hline 6 & Aspergillus nidulans & 6 \\
\hline 7 & Alternaria species & 4 \\
\hline 8 & Fusarium oxysporum & 22 \\
\hline 9 & Fusarium species & 5 \\
\hline 10 & Fusarium semitectum & 2 \\
\hline 11 & $\begin{array}{l}\text { Helminthosporum } \\
\text { species }\end{array}$ & 6 \\
\hline 12 & Mortierella species & 2 \\
\hline 13 & Rhizopus stolonifer & 2 \\
\hline 14 & Sclerotium species & 2 \\
\hline 15 & Tricoderm aviride & 4 \\
\hline 16 & Tricoderma harzianum & 5 \\
\hline & & \\
\hline
\end{tabular}

Table-2: Antagonistic and associative relationship between Fusarium oxysporum and other phyllosphere and fruit fungi of banana

\begin{tabular}{|c|l|c|c|c|c|}
\hline $\begin{array}{l}\text { Sr } \\
\text { No }\end{array}$ & Name of fungi & \multicolumn{4}{|c|}{ Reactions number } \\
\hline & $\begin{array}{l}\text { Fusarium oxysporum } \\
\text { v/s fungi }\end{array}$ & A & B & C & D \\
\hline 1 & Aspergillus flavus & + & - & - & - \\
\hline 2 & Aspergillu sniger & + & - & - & - \\
\hline 3 & Aspergillus fumigatus & + & - & - & - \\
\hline 4 & Aspergillus terreus & + & - & - & - \\
\hline
\end{tabular}




\begin{tabular}{|c|l|c|c|c|c|}
\hline 5 & Aspergillus carbonarius & + & - & - & - \\
\hline 6 & Aspergillu snidulans & + & - & - & - \\
\hline 7 & Alternaria species & - & + & - & - \\
\hline 8 & Fusarium species & - & + & - & - \\
\hline 9 & Fusarium semitectum & + & + & - & - \\
\hline 10 & $\begin{array}{l}\text { Helminthosporum } \\
\text { species }\end{array}$ & + & - & - & - \\
\hline 11 & Mortierella species & - & - & - & - \\
\hline 12 & Rhizopus stolonifer & - & - & - & - \\
\hline 13 & Sclerotium species & - & + & - & - \\
\hline 14 & Tricoderma viride & - & - & + & + \\
\hline 15 & Tricoderma harzianum & - & - & + & + \\
\hline
\end{tabular}

$A=$ Both colonies grow side by side without harming each other

$\mathrm{B}=$ Fusarium oxysporum overlaps on other fungi

$\mathrm{C}=$ Other fungi overlaps on Fusarium oxysporum

$\mathrm{D}=$ Other fungi antagonistic to Fusarium oxysporum

Table-3: Inhibitory action of fungal culture filtrate against Fusarium oxysporum (EMSFO4) on agar plates.

\begin{tabular}{|c|l|c|}
\hline Sr.No. & Fungal culture filtrate & $\begin{array}{c}\text { Inhibitory } \\
\text { zone }(\mathrm{mm})\end{array}$ \\
\hline 1 & Tricoderma viride & 8.0 \\
\hline $\mathbf{2}$ & Tricoderma harzianum & 10.0 \\
\hline
\end{tabular}

Table-4: Percentage control efficacy of Tricoderma harzianum against benomyl resistant mutant (EMS -FO4) of Fusarium oxysporum on banana fruit.

\begin{tabular}{|c|l|l|}
\hline Sr. No. & \multicolumn{1}{|c|}{ Fungal source } & PCE \\
\hline 1 & Culturefiltrate & 80.2 \\
\hline $\mathbf{2}$ & Dualculture & 60.0 \\
\hline 3 & Fungal spore suspension & 65.0 \\
\hline
\end{tabular}

\section{Discussion:}

Integrated management of benomyl resistant Fusarium oxysporumstrain was studied both in vivo and vitro. Tricoderma species are one of the most promising biocontrol agents against fungal plant pathogens.Antibiosis is the generally recognized principal mechanism of interference completion by which fungi may exclude other organism from resources available to each other (Gomathy and Ambikapathy, 2011)Biological control indicated that use of Trichoderma harzianumand Trichoderma viride could increase the percentage control against pathogen. Biological control of plant pathogens has been considered a more natural and ecofriendly control measure. Trichoderma harzianum and Tricoderma piluliferum showed strong antagonism to Alternaria alternata.(Shikha Thakur and N. S. K. Haresh (2016).

\section{Conclusion :}

The study has revealed that there are various associative and antagonistic effect between fungi. Result shows that Aspergillus flavus, Aspergillus species did not show any harmful effect against each other .Resistant mutant ( Ems Fo4) of Fusarium oxysporum overlapped on Aspergillus species and Fusarium species. Where as Trichoderma viride and Trichoderma harzianum showed antagonistic zone towards Fusarium oxysporum. Effect of Trichoderma harzianum culture filtrate on resistant mutant of Fusarium oxysporum was observed by zone inhibition, inhibitatary zone shows that fungal culture filtrate was useful for the management of pathogen.

\section{References :}

Gangawane L. V. and R. S. Saler (1981) Resistance to fungicides in A. flavus. Neth. J. Plant Path.87:254

Gomathy S and Ambikapathy V. (2011) Antagonistic activity of fungi against Pythium debaryanum isolated from chilli field soil. J. Advancs in ap. Sci Res.2 (4) : 291-297

Mukhopadhy A. N. (1995). Exploitation of Gliocladium virens and Trichoderma harzianum for biological seed treatment against soil borne disease. Ind. J. Myco. Pl. Path. 25:124.

Shikha Thakur and N. S. K. Harsh (2016) Biocontrol potential of phylloplane fungi against Alternaria alternata causing leaf spot in Rauwolfia serpentine in vitro. International Journal Of Scientific Research, volume 5:14-16

Shereshta S.M. and A. N. Mukhopadhyay (1992) Biological control of Sclecrotium rolfsii and Rhizoctonia solani in lentil with Gliocladium virens, Ind. Phytopath. Suppl.45:58 